\title{
Deep ultraviolet photoluminescence of Tm-doped AIGaN alloys
}

\author{
N. Nepal, ${ }^{1, a)}$ J. M. Zavada, ${ }^{1}$ D. S. Lee, ${ }^{2}$ A. J. Steckl, ${ }^{2}$ A. Sedhain, ${ }^{3}$ J. Y. Lin, ${ }^{3}$ and \\ H. X. Jiang ${ }^{3}$ \\ ${ }^{1}$ Department of Electrical and Computer Engineering, North Carolina State University, Raleigh, \\ North Carolina 27695, USA \\ ${ }^{2}$ Nanoelectronics Laboratory, University of Cincinnati, Cincinnati, Ohio 45221, USA \\ ${ }^{3}$ Department of Electrical and Computer Engineering and Nano Tech Center, Texas Tech University, \\ Lubbock, Texas 79409, USA
}

(Received 28 November 2008; accepted 20 February 2009; published online 16 March 2009)

\begin{abstract}
The ultraviolet (UV) photoluminescence (PL) properties of Tm-doped $\mathrm{Al}_{x} \mathrm{Ga}_{1-x} \mathrm{~N}(0.39 \leq x \leq 1)$ alloys grown by solid-source molecular beam epitaxy were probed using above-bandgap excitation from a laser source at $197 \mathrm{~nm}$. The PL spectra show dominant UV emissions at 298 and $358 \mathrm{~nm}$ only for samples with $x=1$ and 0.81 . Temperature dependence of the PL intensities of these emission lines reveals exciton binding energies of 150 and $57 \mathrm{meV}$, respectively. The quenching of these UV emissions appears related to the thermal activation of the excitons bound to rare-earth structured isovalent (RESI) charge traps, which transfer excitonic energy to $\mathrm{Tm}^{3+}$ ions resulting in the UV emissions. A model of the RESI trap levels in AlGaN alloys is presented. () 2009 American Institute of Physics. [DOI: 10.1063/1.3097808]
\end{abstract}

AlGaN alloys are large direct band gap, chemically inert compound semiconductor materials that have come under intense scrutiny for optoelectronic device applications. Their high thermal conductivity, large dielectric constant and piezoelectric coefficient, and low electron affinity also make them unique materials for x-ray production, surface acoustic wave devices, cold cathode application, and transistor gate insulation. ${ }^{1-4}$ The wide band gap, which can be tailored from 3.4 to $6.1 \mathrm{eV}$ with $\mathrm{Al}$ content, offers reliable high-voltage/ high-temperature electronic properties as well as optical transparency over a wide spectral range, from the infrared (IR) to the ultraviolet (UV). Recent studies have also focused AlGaN alloys as hosts for rare-earth (RE) ions. ${ }^{5-10}$ Trivalent $\mathrm{RE}$ ions in $\mathrm{AlGaN}$ alloys have been shown to emit narrow intra- $4 f$ transitions over the entire IR to UV spectral range. These sharp emissions, due to well-shielded $4 f$ electrons, have potential applications in color displays, white lighting, remote sensing, and optical communication systems provided the emission efficiency can be improved. The wide band gap $\mathrm{AlGaN}$ alloys provide lower thermal quenching of the RE intra- $4 f$ transitions making room temperature operation possible. ${ }^{11}$ Also, unpaired $4 f$ electrons of RE ions can align along an easy axis resulting in magnetic properties for these RE-doped semiconductors. ${ }^{12,13}$ Thulium is a RE element which has special optical and magnetic characteristics. Tm-doped $\mathrm{Al}_{x} \mathrm{Ga}_{1-x} \mathrm{~N}$ alloys (AlGaN:Tm) have yielded intra- $4 f$ emissions at visible, IR, and UV wavelengths. In addition, such alloys (with $x>0$ ) show room temperature ferromagnetism from the two unpaired $4 f$ electrons. ${ }^{14}$ Therefore $\mathrm{AlGaN}: \mathrm{Tm}$ is a potential material for electrical, magnetic, and optical functionality on a single chip.

Significant research has been carried out on intra- $4 f$ shell optical excitation mechanisms of RE-doped GaN. Song et al. ${ }^{15}$ studied Er- and Pr-implanted GaN by deep level transient spectroscopy. They found RE-related deep charge traps at 0.2 and $0.4 \mathrm{eV}$ below the conduction band (CB) and

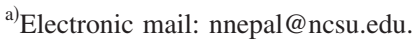

assigned the $0.2 \mathrm{eV}$ trap to a $\mathrm{RE}_{\mathrm{Ga}}-V_{N}$ complex. The studies by $\mathrm{Li}$ et al. ${ }^{16}$ of Eu-doped GaN grown by molecular beam epitaxy (MBE) show a Eu-related charge trap at $0.37 \mathrm{eV}$ below the CB. The intensity of red emission (at $622 \mathrm{~nm}$ ) was directly correlated with this trap. In GaN:Er films grown by metal organic chemical vapor deposition Ugolini et al. ${ }^{17}$ found a charge transfer band at $0.2 \mathrm{eV}$ below the $\mathrm{CB}$, which results in efficient $1.54 \mu \mathrm{m}$ emission. Enhancement of $1.54 \mu \mathrm{m}$ emission from Er-implanted GaN via a violet photoluminescence excitation band has also been reported by Kim et al. ${ }^{18}$

Dorenbos and Van der Kolk ${ }^{19}$ developed a method to establish the $4 f$ ground state energy for divalent and trivalent lanthanide ions relative to the valence and $\mathrm{CBs}$ in $\mathrm{AlGaN}$ alloys. Their model predicts the charge transfer band is located at $\sim 1.44 \mathrm{eV}$ below the $\mathrm{CB}$ in AlN:Tm. A kinetic model of energy transfer from the host lattice to the localized core excited state of RE structured isovalent (RESI) traps has been proposed by Lozykowski. ${ }^{20}$ According to this model the energy-transfer process occurs either through an auger mechanism or from the exciton bound by the RESI trap to the RE core states. Trap energies of 0.72 and $1.41 \mathrm{eV}$ have been identified from the thermal quenching of the cathodoluminescence intensities in Tm-implanted AlN films. ${ }^{21}$

Lee and Steckl ${ }^{22}$ reported on electroluminescence of $\mathrm{Al}_{x} \mathrm{Ga}_{1-x} \mathrm{~N}$ : Tm thin films $(0 \leq x \leq 1)$ grown by MBE. They observed strong blue emissions at 466 and $475 \mathrm{~nm}$ and IR emission at $1420 \mathrm{~nm}$. The intensity of these emissions increased with higher $\mathrm{Al}$ content and peaked at $x \cong 0.8$. Using a laser source at $250 \mathrm{~nm}$, Hömmerich et al. ${ }^{23}$ measured the PL spectra of the same set of samples and found a similar behavior of the blue emission. Since the band gap of the $\mathrm{Al}_{x} \mathrm{Ga}_{1-x} \mathrm{~N}$ alloys increases with higher $\mathrm{Al}$ content, they were able to observe additional transitions in the UV region. They reported a broad emission band near $300 \mathrm{~nm}$ for alloys with $x>0.81$. Nepal et $a l .{ }^{24}$ examined the UV emission from AlN:Tm samples using above-bandgap excitation at $197 \mathrm{~nm}$ and found sharp emission lines at 298 and $356 \mathrm{~nm}$, as seen in Fig. 1(a). These emissions are attributed to the intra- $4 f$ 


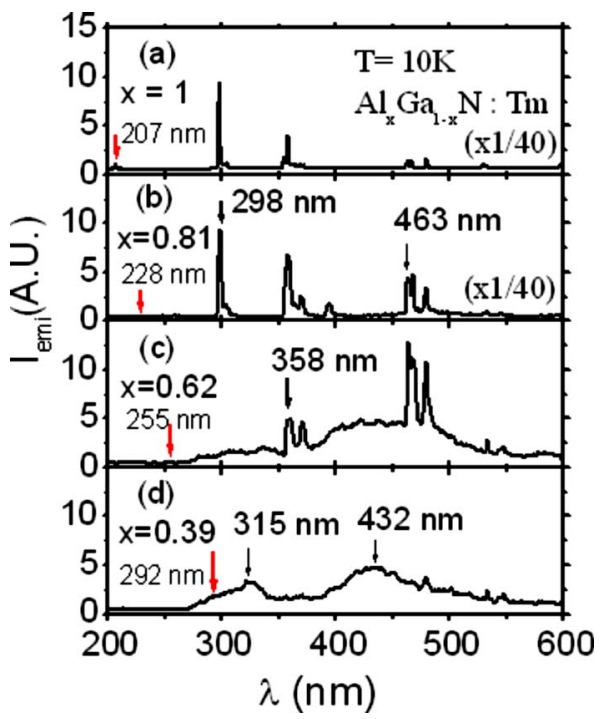

FIG. 1. (Color online) The $10 \mathrm{~K}$ PL spectra of Tm-doped $\mathrm{Al}_{x} \mathrm{Ga}_{1-x} \mathrm{~N}$ $(0.39 \leq x \leq 1)$ alloys with laser excitation at $\lambda_{\text {exc }}=197 \mathrm{~nm}$. Respective band gaps are indicated by thick solid (red) arrows in each panel.

transitions from ${ }^{1} I_{6}$ to ${ }^{3} H_{6}$ and ${ }^{3} F_{4}$ levels, respectively. From the thermal quenching of the emission at $298 \mathrm{~nm}$, they were able to deduce the presence of a RESI charge trap at $1.50 \mathrm{eV}$ below the $\mathrm{CB}$ in close agreement to the predicted value. ${ }^{19}$

In this work, we report on the deep UV PL dynamics of $\mathrm{Al}_{x} \mathrm{Ga}_{1-x} \mathrm{~N}$ :Tm thin films for $0.39 \leq x \leq 1$ using abovebandgap excitation at $197 \mathrm{~nm}$. The temperature dependence of these emissions is used to construct a model for the energy level of the RESI traps over the entire range of $\mathrm{Al}$ content $x$.

The $\mathrm{Al}_{x} \mathrm{Ga}_{1-x} \mathrm{~N}: \operatorname{Tm}(0.39 \leq x \leq 1)$ thin films of $0.5 \mu \mathrm{m}$ thickness were grown on $p$-type $\mathrm{Si}$ (111) substrates by solidsource MBE using elemental $\mathrm{Al}$ and $\mathrm{Tm}$ sources in conjunction with an rf-plasma source supplying atomic $\mathrm{N}^{22}$ The Tm concentration was between 0.2 and 0.5 at. \%. All films were capped with an undoped AlGaN layer. No indication of the formation of second phases was found based on x-ray diffraction measurements. The AlGaN:Tm films were characterized by deep UV PL spectroscopy using above-bandgap excitation from a frequency quadrupled Ti:sapphire laser source.

Figure 1 shows the low temperature PL spectra of $\mathrm{Al}_{x} \mathrm{Ga}_{1-x} \mathrm{~N}: \mathrm{Tm}(0.39 \leq x \leq 1)$ under $197 \mathrm{~nm}$ excitation. The locations of the band edge of the different alloys are indicated by the thick solid (red) arrow in each panel. Prominent emission lines were observed at 298 and $358 \mathrm{~nm}$ for AlN:Tm, as shown in Fig. 1(a). Several weaker lines appear at $463,467,480,529$, and $596 \mathrm{~nm}$. The PL spectrum for $\mathrm{Al}_{x} \mathrm{Ga}_{1-x} \mathrm{~N}$ :Tm film with $x=0.81$ [Fig. 1(b)] is similar, with some broadening of the emissions near 358 and $463 \mathrm{~nm}$. However, for lower $\mathrm{Al}$ content films the PL signal intensity is much weaker. In addition, no emission at $298 \mathrm{~nm}$ is observed even though the band gap of these alloys should permit emission at this wavelength. As shown in Fig. 1(c) the $\mathrm{Al}_{x} \mathrm{Ga}_{1-x} \mathrm{~N}$ :Tm alloy with $x=0.62$ does show distinct UV emission lines near 358 and $465 \mathrm{~nm}$ superimposed on a broad band luminescence. For $x=0.39$ [Fig. 1(d)] only broad PL emission bands were observed.

Figure 2 shows the temperature evolution of the PL spectra of $\mathrm{Al}_{0.81} \mathrm{Ga}_{0.19} \mathrm{~N}$ : Tm over the temperature range from 10 to $250 \mathrm{~K}$. The PL emission intensity of the UV transition at 298 , as well as that of multiple peaks near $358 \mathrm{~nm}$, decreases

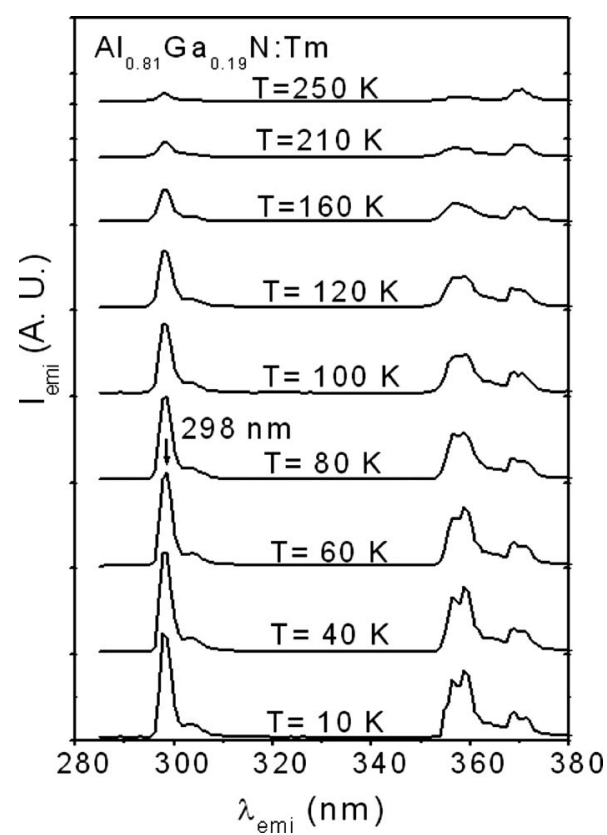

FIG. 2. The temperature evolution of the PL spectra of $\mathrm{Al}_{0.81} \mathrm{Ga}_{0.19} \mathrm{~N}$ : Tm measured from $10-250 \mathrm{~K}$ for excitation at $\lambda_{\mathrm{exc}}=197 \mathrm{~nm}$.

with increasing temperature. Arrhenius plots of the PL intensities of 298 and $358 \mathrm{~nm}$ emission lines that were measured in $\mathrm{Al}_{0.81} \mathrm{Ga}_{0.19} \mathrm{~N}$ : Tm alloy are shown in Figs. 3. The solid lines are the least-squares fit of the data using equation

$$
I_{\mathrm{emi}}=\frac{I_{0}}{1+c e^{-E_{0} / k T}},
$$

where $c$ is a fitting parameter, $k$ is the Boltzmann constant, $T$ is the sample temperature, and $E_{0}$ is the activation energy of the thermal quenching. The best fit values for $E_{0}$ are $59 \pm 4$ and $56 \pm 6 \mathrm{meV}$ for the 298 and $358 \mathrm{~nm}$, respectively.

The size and electronegativity difference between $\mathrm{Tm}^{3+}$ ions and the host cations ( $\mathrm{Al}$ or $\mathrm{Ga}$ ) that they replace create a short range potential and generate RESI charge traps. The RESI trap can capture individual free carriers or a free exciton forming a bound exciton (BX). ${ }^{18,20,21}$ With increasing temperature, the BX will either dissociate or will transfer

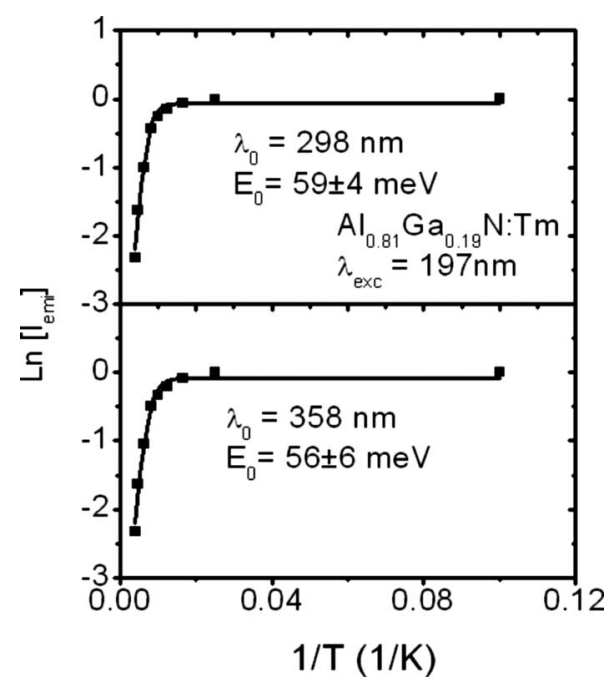

FIG. 3. Arrhenius plots of the integrated PL intensity of (a) $298 \mathrm{~nm}$ and (b) $358 \mathrm{~nm}$ emissions for $\mathrm{Al}_{0.81} \mathrm{Ga}_{0.19} \mathrm{~N}$ : Tm alloy in the temperature range between 10 to $250 \mathrm{~K}$. The solid lines are best fits to the experimental data. The fitted values of the activation energy are indicated in the panels. 


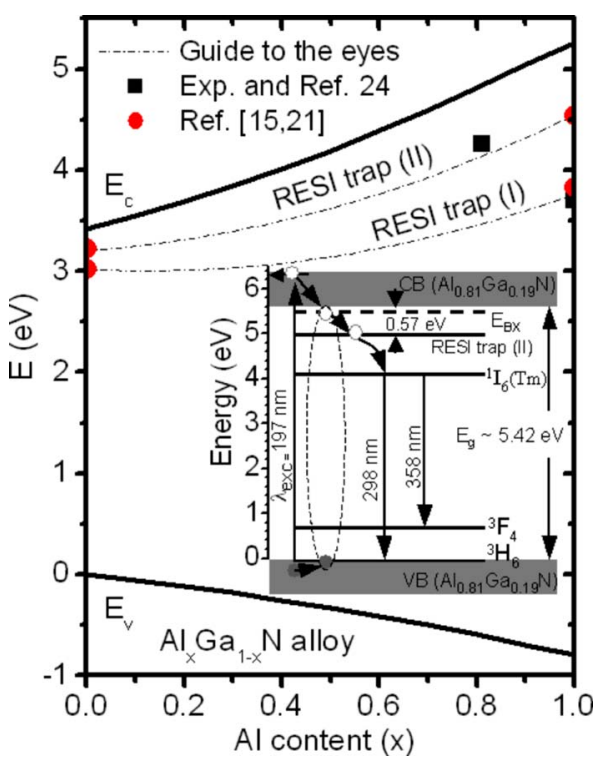

FIG. 4. (Color online) Schematic diagram of the RESI trap levels in $\mathrm{Al}_{x} \mathrm{Ga}_{1-x} \mathrm{~N}$ :Tm alloys as functions of $\mathrm{Al}$ content $x$. The black squares are obtained from the present experiment and Ref. 24. The red circles are data from Refs. 15 and 21. Dotted lines are guides to the eyes for RESI traps (I) and (II). The inset shows the excitation process involving the RESI trap (II) and the ${ }^{1} I_{6},{ }^{3} F_{4}$, and ${ }^{3} H_{6}$ states of a $\mathrm{Tm}^{3+}$ ion in $\mathrm{Al}_{0.81} \mathrm{Ga}_{0.19} \mathrm{~N}$ alloy.

energy to the lower level thereby decreasing the PL emission intensity. The energy transfer process from the RESI BX to the $4 f$ state of $\mathrm{Tm}^{3+}$ ions has been discussed elsewhere. ${ }^{19-21}$ The thermal quenching of the PL intensities of the UV transitions at 298 and $358 \mathrm{~nm}$ in the $\mathrm{Al}_{0.81} \mathrm{Ga}_{0.19} \mathrm{~N}$ : Tm alloy results to an average activation energy $E_{0}$ of $\sim 57 \mathrm{meV}$. Consequently, from Haynes' rule, ${ }^{25}$ the expected energy level of the RESI trap is $\sim 0.57 \mathrm{eV}$ below the $\mathrm{CB}$ in $\mathrm{Al}_{0.81} \mathrm{Ga}_{0.19} \mathrm{~N}$ :Tm alloy. Previously, we reported that the measured energy level of the RESI trap was $1.50 \mathrm{eV}$ in AlN:Tm. ${ }^{24}$ Thus, the thermal quenching of the deep UV transitions in $\mathrm{Al}_{x} \mathrm{Ga}_{1-x} \mathrm{~N}$ :Tm is due to thermal dissociation of the excitons bound to RESI charge traps at 1.50 and $0.57 \mathrm{eV}$ for alloys with $x=1$ and 0.81 , respectively.

From the present data and previous experimental results we have constructed a schematic energy level model, as shown in Fig. 4, for the RESI trap levels in $\mathrm{Al}_{x} \mathrm{Ga}_{1-x} \mathrm{~N}$ alloys covering the entire range of $\mathrm{Al}$ content $x$. We have taken the low temperature bandgap of $\mathrm{AlN}$ and $\mathrm{GaN}$ to be 6.10 and 3.4 $\mathrm{eV}$, respectively. The band offset of the AlGaN alloy system was taken as $70 \%$ for the CB and $30 \%$ for the VB. The RESI trap (I) at $1.50 \mathrm{eV}$ in AlN:Tm and RESI trap (II) at $0.57 \mathrm{eV}$ in $\mathrm{Al}_{0.81} \mathrm{Ga}_{0.19} \mathrm{~N}$ are indicated by black squares. Results from Er- and Pr-implanted GaN (Ref. 15) and Tm-implanted AlN (Ref. 21) are shown as filled red circles. The dotted lines are guides to the eyes for RESI traps (I) and (II). Consistent with previous studies, our experiments have determined two different RESI charge traps in AlGaN alloys that are responsible for $\mathrm{Tm}^{3+}$ related deep UV emissions. The inset of Fig. 4 shows an energy level diagram involving ${ }^{1} I_{6},{ }^{3} F_{4}$, and ${ }^{3} H_{6}$ states of a $\mathrm{Tm}^{3+}$ ion in the $\mathrm{Al}_{0.81} \mathrm{Ga}_{0.19} \mathrm{~N}$ alloy. It also shows the deep intra- $4 f$ shell UV emissions and the associated excitation paths. We have taken the low temperature bandgap of $\mathrm{Al}_{0.81} \mathrm{Ga}_{0.19} \mathrm{~N} / \mathrm{Si}$ to be $5.42 \mathrm{eV}$ and have placed the $\mathrm{BX}$ level and the RESI trap (II) at $\sim 57 \mathrm{meV}$ and $0.57 \mathrm{eV}$ below the $\mathrm{Al}_{0.81} \mathrm{Ga}_{0.19} \mathrm{~N} \mathrm{CB}$, respectively. The $\mathrm{UV}$ excitation at $197 \mathrm{~nm}$ moves an electron from the VB to within the CB. With nonradiative relaxation, the electron moves to the band edge and forms a BX. Subsequently, the BX transfers energy to the RESI trap (II) and excites the ${ }^{1} I_{6}$ level of a $\mathrm{Tm}^{3+}$ ion. Intra- $4 f$ transitions result in emissions at 298 and $358 \mathrm{~nm}$.

In summary, we have observed dominant deep UV PL emissions from Tm-doped $\mathrm{Al}_{x} \mathrm{Ga}_{1-x} \mathrm{~N}(x=1$ and 0.81$)$ alloys using a laser source at $197 \mathrm{~nm}$. The temperature dependence of the PL intensities of these emission lines indicates RESI charge traps at energies of 1.50 and $0.57 \mathrm{eV}$ below the CB. The quenching of the UV emission in AlGaN:Tm alloys is controlled by the thermal activation of the excitons bound to these RESI charge traps. Based on the data, we have developed a schematic model for the two RESI trap energy levels over the entire range of $x$ in Tm-doped AlGaN alloys.

This work was supported in part by the U. S. Army Research Office Grant No. W911NF-06-1-0296(UN). N.N. is fellow of NRC Postdoctoral Associateship Program.

${ }^{1}$ T. Matsutani, M. Kiuchi, K. Shirouzu, A. Yoshioka, R. Shimizu, and S. Takahashi, Solid State Phenom. 107, 43 (2005).

${ }^{2}$ V. Mortet, O. Elmazria, M. Nesladek, M. B. Assouar, G. Vanhoyland, J. D'Haen, M. D'Olieslaeger, and P. Alnot, Appl. Phys. Lett. 81, 1720 (2002).

${ }^{3}$ A. T. Sowers, J. A. Christman, M. D. Bremser, B. L. Ward, R. F. Davis, and R. J. Nemanich, Appl. Phys. Lett. 71, 2289 (1997).

${ }^{4}$ M. M. De Souza, S. Jejurikar, and K. P. Adhi, Appl. Phys. Lett. 92, 093509 (2008).

${ }^{5}$ A. J. Steckl and J. M. Zavada, MRS Bull. 24, 33 (1999).

${ }^{6}$ H. J. Lozykowski, W. M. Jadwisienczak, and I. Brown, Appl. Phys. Lett. 74, 1129 (1999).

${ }^{7}$ A. J. Steckl, M. Garter, D. S. Lee, J. Heikenfeld, and R. Birkhahn, Appl. Phys. Lett. 75, 2184 (1999).

${ }^{8}$ K. Lorenz, U. Wahl, E. Alves, S. Dalmasso, R. W. Martin, K. P. O'Donnell, S. Ruffenach, and O. Briot, Appl. Phys. Lett. 85, 2712 (2004)

${ }^{9}$ J. M. Zavada, N. Nepal, J. Y. Lin, H. X. Jiang, E. Brown, U. Hömmerich,

J. Hite, G. T. Thaler, C. R. Abernathy, S. J. Pearton, and R. Gwilliam, Appl. Phys. Lett. 89, 152107 (2006).

${ }^{10}$ A. J. Steckl, J. H. Park, and J. M. Zavada, Mater. Today 10, 20 (2007).

${ }^{11}$ J. D. MacKenzie, C. R. Abernathy, S. J. Pearton, U. Hömmerich, X. Wu, R. N. Schwartz, R. G. Wilson, and J. M. Zavada, Appl. Phys. Lett. 69, 2083 (1996) and references therein.

${ }^{12}$ S. Dhar, O. Brandt, M. Ramsteiner, V. F. Sapega, and K. H. Ploog, Phys. Rev. Lett. 94, 037205 (2005)

${ }^{13}$ J. M. Zavada, N. Nepal, C. Ugolini, J. Y. Lin, H. X. Jiang, R. Davies, J. Hite, C. R. Abernathy, J. Pearton, E. E. Brown, and U. Hommerich, Appl. Phys. Lett. 91, 054106 (2007).

${ }^{14}$ N. Nepal, S. M. Bedair, N. A. El-Masry, D. S. Lee, A. J. Steckl, and J. M. Zavada, Appl. Phys. Lett. 91, 222503 (2007).

${ }^{15}$ S. F. Song, W. D. Chen, C. Zhang, L. Bian, C. C. Hsu, L. W. Lu, Y. H. Zhang, and J. Zhu, Appl. Phys. Lett. 86, 152111 (2005).

${ }^{16} \mathrm{Z}$. Li, H. Bang, G. Piao, J. Sawahata, and K. Akimoto, J. Cryst. Growth 240, 382 (2002).

${ }^{17}$ C. Ugolini, N. Nepal, J. Y. Lin, H. X. Jiang, and J. M. Zavada, Appl. Phys. Lett. 90, 051110 (2007).

${ }^{18}$ S. Kim, S. J. Rhee, X. Li, J. J. Coleman, and S. G. Bishop, Appl. Phys. Lett. 76, 2403 (2000).

${ }^{19}$ P. Dorenbos and E. Van der Kolk, Opt. Mater. 30, 1052 (2008).

${ }^{20}$ H. J. Lozykowski, Phys. Rev. B 48, 17758 (1993).

${ }^{21}$ H. J. Lozykowski and W. M. Jadwisienczak, Phys. Status Solidi B 244, 2109 (2007)

${ }^{22}$ D. S. Lee and A. J. Steckl, Appl. Phys. Lett. 83, 2094 (2003).

${ }^{23}$ U. Hömmerich, E. E. Nyein, D. S. Lee, A. J. Steckl, and J. M. Zavada, Appl. Phys. Lett. 83, 4556 (2003).

${ }^{24}$ N. Nepal, J. M. Zavada, D. S. Lee, and A. J. Steckl, Appl. Phys. Lett. 93, 061110 (2008).

${ }^{25}$ J. R. Haynes, Phys. Rev. Lett. 4, 361 (1960). 